\title{
Pulmonary type-2 innate lymphoid cells in paediatric severe asthma: phenotype and response to steroids
}

\author{
Prasad Nagakumar ${ }^{1,2,3}$, Franz Puttur (1) ${ }^{1,3}$, Lisa G. Gregory ${ }^{1}$, Laura Denney ${ }^{1}$, \\ Louise Fleming $\mathbb{1}^{2}$, Andrew Bush ${ }^{2}$, Clare M. Lloyd ${ }^{1,4}$ and Sejal Saglani ${ }^{1,2,4}$ \\ Affiliations: ${ }^{1}$ National Heart and Lung Institute, Imperial College London, London, UK. ${ }^{2}$ Respiratory \\ Paediatrics, Royal Brompton Hospital and National Heart and Lung Institute, Imperial College London, \\ London, UK. ${ }^{3}$ Both authors contributed equally. ${ }^{4}$ Both authors contributed equally.
}

Correspondence: Sejal Saglani, Inflammation, Repair and Development, National Heart and Lung Institute, Sir Alexander Fleming Building, Imperial College London, Exhibition Road, London, SW7 2AZ, UK. E-mail: s.saglanidimperial.ac.uk

@ERSpublications

Children with severe asthma have a distinct type-2 airway molecular phenotype with higher ILC2s, Th2 cells and eosinophils than difficult asthma, while IL-17 $7^{+}$cells are similar. ILC2s are sensitive to systemic steroids whereas IL- $17^{+}$cells are unchanged. bit.ly/2JMtW1R

Cite this article as: Nagakumar P, Puttur F, Gregory LG, et al. Pulmonary type-2 innate lymphoid cells in paediatric severe asthma: phenotype and response to steroids. Eur Respir J 2019; 54: 1801809 [https://doi. org/10.1183/13993003.01809-2018].

ABSTRACT Children with severe therapy-resistant asthma (STRA) have poor control despite maximal treatment, while those with difficult asthma (DA) have poor control from failure to implement basic management, including adherence to therapy. Although recognised as clinically distinct, the airway molecular phenotype, including the role of innate lymphoid cells (ILCs) and their response to steroids in DA and STRA is unknown.

Immunophenotyping of sputum and blood ILCs and T-cells from STRA, DA and non-asthmatic controls was undertaken. Leukocytes were analysed longitudinally pre- and post-intramuscular triamcinolone in children with STRA. Cultured ILCs were evaluated to assess steroid responsiveness in vitro.

Airway eosinophils, type 2 T-helper (Th2) cells and ILC2s were significantly higher in STRA patients compared to DA and disease controls, while IL- $17^{+}$lymphoid cells were similar. ILC2s and Th2 cells were significantly reduced in vivo following intramuscular triamcinolone and in vitro with steroids. Furthermore, asthma attacks and symptoms reduced after systemic steroids despite persistence of steroidresistant IL- $17^{+}$cells and eosinophils.

Paediatric STRA and DA have distinct airway molecular phenotypes with STRA characterised by elevated type-2 cells. Systemic corticosteroids, but not maintenance inhaled steroids resulted in improved symptom control and exacerbations concomitant with a reduction in functional ILC2s despite persistently elevated IL- $17^{+}$lymphoid cells. 


\section{Introduction}

Paediatric severe therapy-resistant asthma (STRA) is characterised by persistent poor control despite maximal doses of treatment and optimal assessment of modifiable factors such as adherence, allergen and smoke exposure. STRA affects $\sim 2 \%$ of children with asthma, but results in significant morbidity [1], utilising up to $50 \%$ of all healthcare resources for asthma [2, 3]. Difficult asthma (DA) in children is characterised by poor control despite maximal prescribed therapy, but detailed clinical assessments reveal modifiable factors, most commonly lack of adherence to maintenance therapy, as a reason for the apparent poor control [4,5]. We have shown that paediatric DA and STRA have distinct clinical phenotypes [6]. In contrast to STRA, when the basics of asthma management are addressed, children with DA have lower exhaled nitric oxide levels, improved lung function and are able to reduce their daily dose of inhaled corticosteroids while maintaining control. Moreover, DA patients continue to have significantly fewer exacerbations than STRA up to 6 years later [6]. This suggests that children with DA have steroid-sensitive disease, while STRA patients have disease that is resistant to maximal maintenance corticosteroids. As a group, children with STRA have reduced lung function, marked eosinophilic airway inflammation and airway remodelling [1]. However, little is known about the molecular phenotype of DA, and whether DA and STRA can be distinguished using molecular as well as clinical phenotypes. If this is possible, it may prevent inappropriate administration of biologicals to children with steroid-sensitive disease.

It is recognised that STRA is a heterogeneous disease [7] and the underlying immunological mechanisms are yet to be fully understood. Although traditionally allergic asthma is considered a type 2 T-helper cell (Th2)-mediated disease, emerging evidence, from studies in adults and children, suggests that non-Th2 mechanisms may contribute, particularly to severe disease $[8,9]$. Interleukin (IL)-33 is an innate epithelial cytokine which is elevated in paediatric STRA and is associated with airway remodelling and severe steroid-resistant disease $[10,11]$. Numerous experimental murine models have underscored the importance of IL-33 in the initiation of allergic airways disease via the induction of type 2 innate lymphoid cells (ILCs) [12-14]. ILCs are a rare population of cells of lymphoid lineage, found predominantly at mucosal surfaces, which can mirror the functions of Th subtypes. Type 2 ILCs are implicated in allergic diseases [15] and are increased in sputum and bronchoalveolar lavage (BAL) from adults [16] and children with severe asthma [17]. Although their importance in severe disease is predicted, little is known regarding their role in milder disease, or how common asthma treatments, such as steroids, impact their function or phenotype.

The Th17 pathway has also been proposed as important in mediating adult, non-type 2 severe asthma [18]. Although IL-17 is induced from paediatric STRA peripheral blood mononuclear cells (PBMCs) following in vitro stimulation with steroids [19], nothing is known about IL-17 ${ }^{+}$ILCs or the functional importance of IL-17 in paediatric STRA.

Since pulmonary IL-33 remains elevated despite maximal steroid therapy in paediatric STRA [10], we hypothesised that the downstream effector cells, type 2 ILCs, remain elevated despite steroids and mediate the pathophysiology of STRA, while they would be lower in DA. We analysed the phenotype and proportion of airway (induced sputum) and peripheral blood $\mathrm{CD}^{+}{ }^{+}$T-cells and ILCs in children with STRA compared to DA and age-matched disease controls. To investigate the effect of steroids in true severe disease, proportions of lymphoid cells were compared in induced sputum before and after systemic steroids, and cultured PBMCs were stimulated with allergen and steroids.

\section{Materials and methods}

Subjects

Children (aged 6-16 years) undergoing clinically indicated investigations (blood tests and induced sputum) for STRA, DA or recurrent lower respiratory tract infections were included. Clinical characterisation, processing of induced sputum and PBMCs were performed as described previously [17]. STRA $(n=16)$ children had confirmed asthma with poor control despite maximal dose inhaled corticosteroids $\left(\geqslant 800 \mu \mathrm{g} \cdot\right.$ day $^{-1}$ budesonide equivalent) and optimisation of underlying modifiable factors, such as adherence [2]. Patients with DA $(n=6)$ were prescribed maximal dose maintenance therapy, but had evidence of poor adherence as an explanation for poor control [20]. Spirometry and bronchodilator reversibility, fractional exhaled nitric oxide, sputum induction and symptom control (asthma control test) were undertaken in all STRA and DA children. Non-asthmatic disease control patients $(n=8)$ had persistent or recurrent cough not responding to antibiotics $(n=6)$, cystic fibrosis $(n=1)$ or primary ciliary dyskinesia $(\mathrm{n}=1)$; these were collectively termed "chronic inflammation".

Study approval was obtained from the local research ethics committee, parental written informed consent and age-appropriate child assent was obtained. 
Flow cytometry

Cells were incubated for $4 \mathrm{~h}$ with phorbol-12-myristate 13-acetate [21], ionomycin and brefeldin A, stained with a fixable viability stain (Zombie UV; BioLegend, London, UK) and ILC and T-cell markers. All ILCs were lineage-negative (CD3, CD14, CD16, CD19, CD20, CD56, CD4, FceR1), CD45 ${ }^{+}$Type 2 ILCs were $\mathrm{CRTH}_{2}{ }^{+}$or $\mathrm{CD} 127^{+}$and/or IL- $13^{+}$or IL- $4^{+}$. Th2 cells were $\mathrm{CD}^{+} \mathrm{CD} 4^{+}$expressing CRTH2/IL-13/IL-4 Th17 cells $\left(\mathrm{CD} 4^{+} \mathrm{IL}-17^{+}\right)$and IL-17 ${ }^{+}$ILCs (lineage-negative, IL-17 ${ }^{+}$). Antibodies used were CD45 (Life Technologies, Paisley, UK), lineage cocktail, CD127, CRTH2, CD3, CD4, CD8, IL-13, IL-17A, GATA-3 (BioLegend) FceR1 (eBioscience, Altringham, UK). Data was acquired on BD Fortessa (BD Bioscience, SanJose, CA, USA) and analysed using Flowjo v10 (Flowjo, Ashland, OR, USA).

PBMC culture

PBMCs were cultured with IL-2 $\left(20 \mathrm{ng} \cdot \mathrm{mL}^{-1}\right)$ (T-cells), or IL-7 (ILCs) $\left(20 \mathrm{ng} \cdot \mathrm{mL}^{-1}\right)$ with $25 \mu \mathrm{g} \cdot \mathrm{mL}^{-1}$ house dust mite (HDM) extract (Greer Laboratories, Lenoir, NC, USA) and/or budesonide $\left(10^{-7} \mathrm{mmol} \cdot \mathrm{L}^{-1}\right)$. Culture supernatants were collected after $72 \mathrm{~h}$ for cytokine analysis.

\section{ILC culture}

ILCs were enriched from whole blood (adult mild asthmatics and healthy controls) using RosetteSep ${ }^{\mathrm{TM}}$ Human ILC2 Enrichment Kit (Stemcell Technologies, Cambridge, UK) and sorted by flow cytometry using $\mathrm{CD}_{4}{ }^{+}$lineage ${ }^{\text {neg }}$ (CD1a, CD3, CD4, CD5, CD8, CD11c, CD14, CD16, CD19, CD20, CD34, FceRI and $\mathrm{CD} 123$ ) (BioLegend), $\mathrm{CD} 161^{+}, \mathrm{CD} 127^{+}, \mathrm{CRTH} 2^{+}$and $\mathrm{C}-\mathrm{Kit}^{\mathrm{var}}$. In order to fully differentiate between lineage negative and positive cells after RosetteSep ${ }^{\mathrm{TM}}$ antibody cocktail staining, an expanded lineage panel was used. ILCs were cultured in IL-2, IL-7 (5 ng. $\left.\mathrm{mL}^{-1}\right)$ and IL-33 (10 ng.mL $\left.{ }^{-1}\right)$ (eBioscience). ILC cultures were stimulated as described for PBMC cultures.

\section{Quantitative PCR}

Cultured ILCs were lysed with $350 \mu \mathrm{L}$ RLT buffer (Qiagen, Manchester, UK). Total RNA was extracted using the RNeasy Micro Kit (Qiagen) and converted to cDNA. Real-time PCRs were performed using Taqman Fast Advanced Master Mix with TaqMan primer/probe sets for IL13 and NR3C1 and data normalised against GAPDH and $\beta$-actin to calculate relative expression.

\section{Immunohistochemistry}

Cytospins of ILC2s stained with anti-Glucocorticoid Receptor (D8H2) XP ${ }^{\star}$ rabbit mAb followed by a biotinylated goat anti-rabbit IgG secondary antibody. Images were acquired on an inverted laser scanning confocal microscope (SP5; Leica Microsystems, Wetzlar, Germany).

\section{Statistics}

Sample size was opportunistic as there were no data to inform a power calculation. Nonparametric Kruskal-Wallis analysis, followed by Dunn's corrections for multiple comparisons, were used to assess between-group differences. Wilcoxon matched pairs test was used for paired data. Data are presented as median. Correlations were assessed using the Spearman rank correlation test and GraphPad Prism v5 (GraphPad Software, La Jolla, CA, USA). Statistical significance was accepted as $\mathrm{p}<0.05$.

\section{Results}

\section{Patient characteristics}

The baseline characteristics of children with STRA, DA and non-asthmatic controls are shown in table 1. Administration of intramuscular triamcinolone was undertaken in STRA as part of our clinical severe asthma investigation protocol and to assess suitability for add-on therapies such as omalizumab [4]. All STRA and DA patients had been prescribed high-dose inhaled steroids, long acting $\beta$-agonists and/or leukotriene receptor antagonists (table 1) [2]. STRA patients had significantly higher total serum IgE and sputum eosinophils compared to DA and non-asthmatic controls. There was no difference in age, weight, height, forced expiratory volume in $1 \mathrm{~s}$ (FEV1), or forced vital capacity between the groups (table 1).

\section{Airway eosinophils were only increased in STRA, while blood eosinophils were higher in STRA and DA compared to controls}

Although elevated blood eosinophils are considered a biomarker for severe asthma in adults [22], their role in paediatric severe asthma is less certain [23]. We compared eosinophil numbers in blood and sputum in STRA, DA and non-asthmatic controls. Blood eosinophils were similarly elevated in both STRA and DA compared to controls, while sputum eosinophils were only significantly higher in STRA, and were almost undetectable in DA and controls (figure 1a). There was no correlation between sputum and blood eosinophils in either children with STRA or DA (supplementary figure S1). Sputum neutrophils were higher in non-asthmatic controls who have recurrent infections (figure 1b), while lymphocytes and 
TABLE 1 Demographics of paediatric severe therapy-resistant asthma (STRA), difficult asthma (DA) and chronic inflammation patients

\begin{tabular}{|c|c|c|c|c|c|}
\hline & STRA & DA & $\begin{array}{c}\text { Chronic } \\
\text { inflammation }\end{array}$ & $\begin{array}{l}\text { p-value (STRA } \\
\text { versus DA) }\end{array}$ & $\begin{array}{l}\text { p-value (STRA versus } \\
\text { chronic inflammation) }\end{array}$ \\
\hline Subjects & 16 & 6 & 8 & & \\
\hline Age years & $12.8(6.9-16.1)$ & $14(8.1-16.5)$ & $9.8(6.1-16.2)$ & 0.2 & 0.05 \\
\hline Age at onset of asthma years & $4.4(2-8)$ & $4.2(2-7)$ & & 0.3 & \\
\hline Male & $17(68)$ & $6(75)$ & $4(44.4)$ & 0.05 & 0.03 \\
\hline Weight kg & $49(23-94.7)$ & $42.5(32.6-80)$ & $48.5(38.2-62.3)$ & 0.07 & 0.1 \\
\hline Height $\mathrm{cm}$ & $150(103-186)$ & $148(55-167)$ & $148(128-161)$ & 0.1 & 0.1 \\
\hline FEV 1 L & $1.84(0.98-4.79)$ & $1.89(1.59-3.08)$ & $1.91(1.4-2.65)$ & 0.07 & 0.05 \\
\hline FEV $1 \%$ & $87.5(66-134)$ & $82.5(63-110)$ & $86(63-115)$ & 0.06 & 0.1 \\
\hline FVC L & $2.9(1.2-4.79)$ & $2.3(2.13-3.76)$ & $2.7(1.5-4)$ & 0.09 & 0.1 \\
\hline FVC $\%$ & $99.8(89.6,132.1)$ & $95(68-106)$ & $102.5(76-124)$ & 0.08 & 0.3 \\
\hline Total IgE IU.mL ${ }^{-1}$ & $321.5(21-1938)$ & $161(81-801)$ & $38.5(19-1252)$ & 0.05 & 0.02 \\
\hline Atopic & $16(100)$ & 5 (83.3) & & 0.4 & \\
\hline $\begin{array}{l}\text { Aeroallergen sensitisation } \\
\text { (number of allergens) }\end{array}$ & $3(1-5)$ & $2(1-3)$ & & 0.1 & \\
\hline $\begin{array}{l}\text { Sum of specific aeroallergen } \\
\operatorname{IgE~IU} \cdot \mathrm{mL}^{-1}\end{array}$ & $3.3(0.8-102)$ & $2.1(0.9-100)$ & & 0.2 & \\
\hline ACT & 21 (13-23) & $16.5(16-23)$ & & 0.04 & \\
\hline FenO ppb & $12.5(8-43)$ & $12(10-31)$ & & 0.1 & \\
\hline ICS mg.day ${ }^{-1}$ & $1(0.8-2)$ & $1(0.8-2)$ & $0.4(0-1.6)$ & 0.1 & 0.04 \\
\hline OCS & 1 & 0 & 0 & & \\
\hline Omalizumab & 5 & 0 & & & \\
\hline Sputum eosinophils \% & $4.7(1-68.1)$ & $0(0-1.2)$ & $0.00(0-0.5)$ & 0.002 & 0.002 \\
\hline Sputum neutrophils \% & $12.5(0-74)$ & $7.7(0-77.3)$ & $71.3(34-97.5)$ & 0.1 & 0.001 \\
\hline Sputum lymphocytes \% & $0(0-1)$ & $0(0-0)$ & $0(0-0)$ & 0.2 & 0.2 \\
\hline
\end{tabular}

Data are presented as $\mathrm{n}$, median (range) or $\mathrm{n}(\%)$, unless otherwise stated. FEV1: forced expiratory volume in 1 s; FVC: forced vital capacity; ACT: Asthma Control Test, FeNO: exhaled nitric oxide fraction; ICS: inhaled corticosteroids; OCS: oral corticosteroids.

macrophages were elevated in STRA and DA (figure 1c,d). Blood neutrophils, monocytes and lymphocytes were similar in all three groups (figure $1 b, c$ and $d$ ).

Increased functional airway type 2 ILCs and T-cells in paediatric STRA

We have previously identified type 2 ILCs and T-cells in BAL and sputum from STRA patients on the basis of expression of the extracellular marker $\mathrm{CRTH} 2^{+}$[17]. However, in order to gain greater phenotypic and functional definition we examined all $\mathrm{Lin}^{\text {neg }} \mathrm{CD} 5^{+}$ILCs for the expression of IL-13, IL-4 and IL-17, the cytokines that may drive disease phenotype (supplementary figure 2). STRA patients had significantly higher frequency of sputum ILCs $\left(\mathrm{Lin}^{\text {neg }} \mathrm{CD} 45^{+}\right.$) and CD4 T-cells expressing CRTH2 than DA and non-asthmatic controls (figure 2a). In addition, a higher frequency of sputum ILCs and CD4 T-cells from STRA patients expressed IL-4 (figure 2b) and IL-13 (figure 2c) compared to DA and non-asthmatic controls. However, there was no difference between the three groups in frequency of sputum ILCs or CD4 T-cells expressing IL-17 (figure 2d). Of note, the non-asthmatic controls with chronic inflammation had elevated sputum neutrophils (figure 1b), but no increase in IL-17 $7^{+}$ILCs or CD4 T-cells (figure 2d). An increased number of ILCs and T-cells with the capacity to produce type 2 cytokines defines the patients with STRA. Therefore, we measured the levels of type 2 cytokines in the sputum of patients. Levels of IL-13 were elevated in STRA patients compared to controls and the amount of IL-5 was significantly increased in these patients (supplementary figure 3). Peripheral blood ILCs and CD4 T-cells expressing CRTH2, IL-13 or IL-17 were not different between STRA and DA (supplementary figure 4a-c).

Phenotypic features of airway IL-13+ ILCs in STRA, DA and controls

Numerous definitions are used for ILCs, based on extracellular expression of CRTH2, or IL-7R $\alpha$ (CD127) or intracellular cytokine expression (IL- $\left.13^{+}, \mathrm{IL}-4^{+}, \mathrm{IL}-17^{+}\right)$. We examined both extracellular markers and intracellular cytokine expression to further define the phenotype of airway ILCs in DA and STRA. Furthermore, in order to determine whether only $\mathrm{CD} 127^{+}$ILCs are steroid resistant in patients with severe asthma, as recently published [24], we investigated $\mathrm{Lin}^{-} \mathrm{CD} 45^{+}$cells that expressed CD127 or CRTH2 or IL-13. Sputum Lin ${ }^{\text {neg }} \mathrm{CD} 45^{+} \mathrm{IL}-13^{+}$cells expressing the type 2 marker CRTH2 were significantly higher in STRA than DA (figure 3a), while $\mathrm{Lin}^{\text {neg }} \mathrm{CD} 45^{+} \mathrm{IL}-13^{+}$cells expressing the general ILC marker CD127 were 
a)

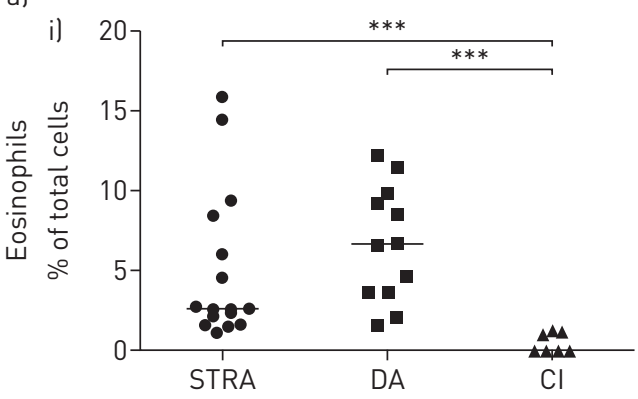

b)

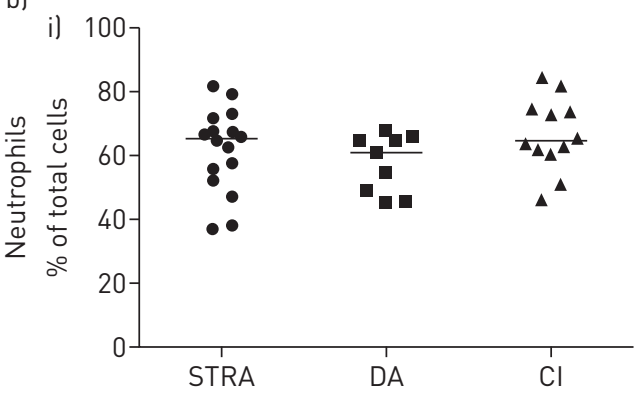

c)

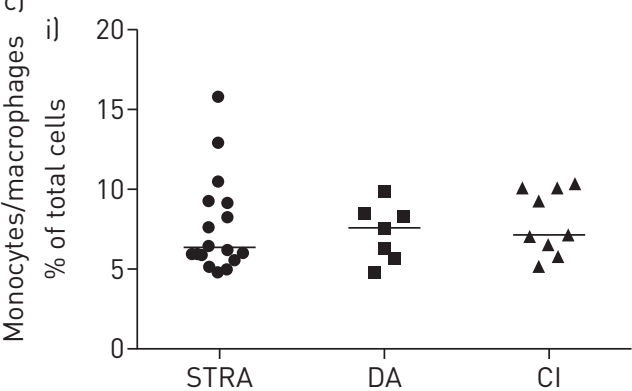

d)

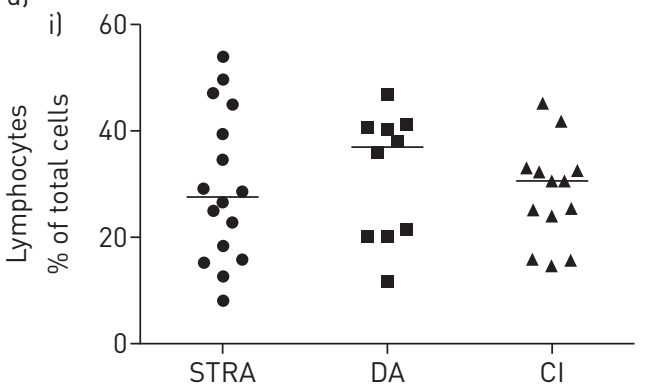

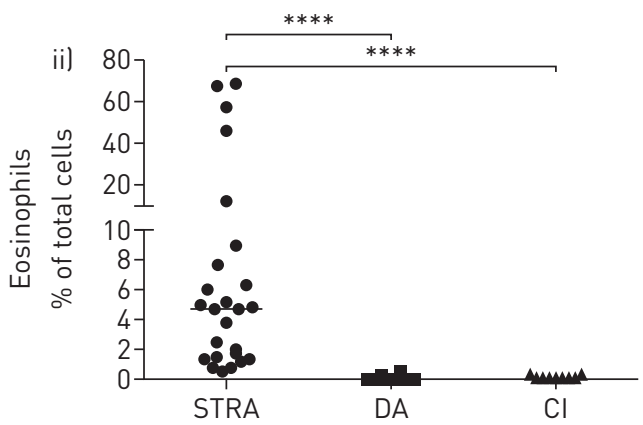
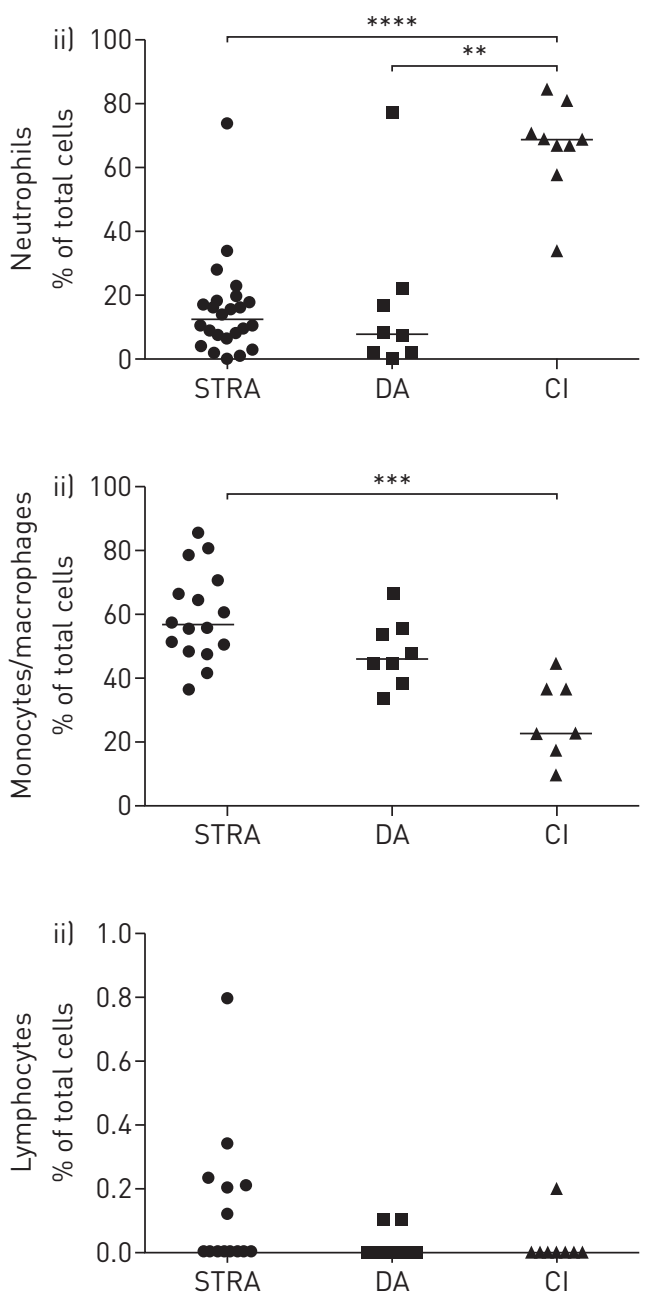

FIGURE 1 Elevated blood eosinophils in severe therapy-resistant asthma (STRA) and difficult asthma (DA) compared to chronic inflammation (CI), but sputum eosinophils only increased in STRA. a) Frequency of eosinophils in i) blood and ii) induced sputum from children with STRA, DA and with recurrent lower respiratory tract infections (CI) assessed by morphology; frequency of b) neutrophils, c) monocytes and macrophages and d) lymphocytes in i) blood and ii) induced sputum. Kruskall-Wallis test with a Dunn's post-test, followed by Mann-Whitney test between indicated pairs of groups. ${ }^{* *}: p<0.01,{ }^{* * *}: p<0.001$ and $* * * *: p<0.0001$. STRA $n \geqslant 16$, DA $n \geqslant 6$ and $\mathrm{CI} n \geqslant 7$.

similar in both groups (figure 3b). Of all sputum Lin $^{\text {neg }}{ }^{2} \mathrm{CD} 45^{+} \mathrm{IL}-13^{+}$cells in STRA, only $16 \%$ were both $\mathrm{CD} 127^{+}$and $\mathrm{CRTH} 2^{+}$, the majority $(65 \%)$ of sputum $\mathrm{Lin}^{\text {neg }} \mathrm{IL} 13^{+}$cells did not express $\mathrm{CD} 127$ and only $33 \%$ expressed CRTH2 (figure 3c). Therefore, we assessed the frequency of $\mathrm{Lin}^{\text {neg }} \mathrm{CD} 45^{+} \mathrm{IL}-13^{+}$that were $\mathrm{CRTH} 2{ }^{+} \mathrm{CD} 127^{+}$and $\mathrm{CRTH} 2^{-} \mathrm{CD} 127^{-}$in STRA compared to DA and controls. Cells that expressed both CD127 and CRTH2 or neither of the markers were increased in STRA compared to controls (figure 3d,e), suggesting that $\mathrm{Lin}^{\text {neg }} \mathrm{CD} 45^{+} \mathrm{IL}-13^{+}$cells may be functionally important in driving STRA. Interestingly, in a 
a)

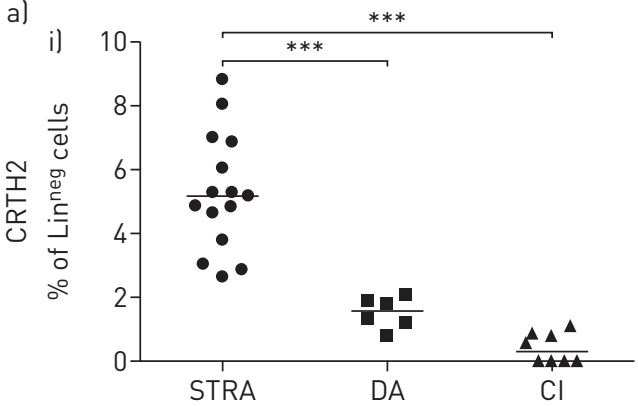

b)

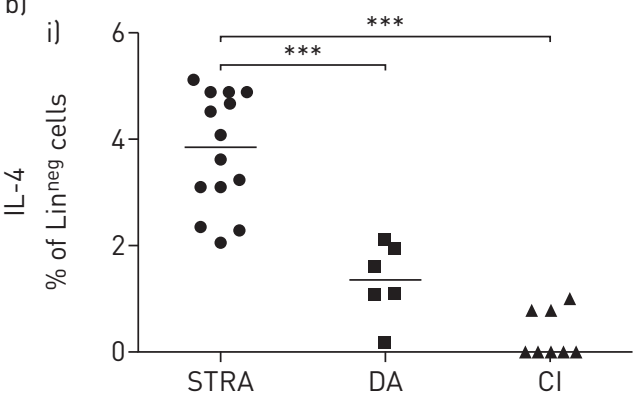

c)

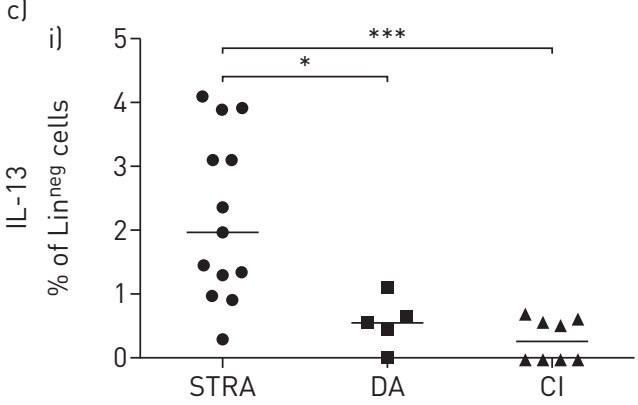

d)

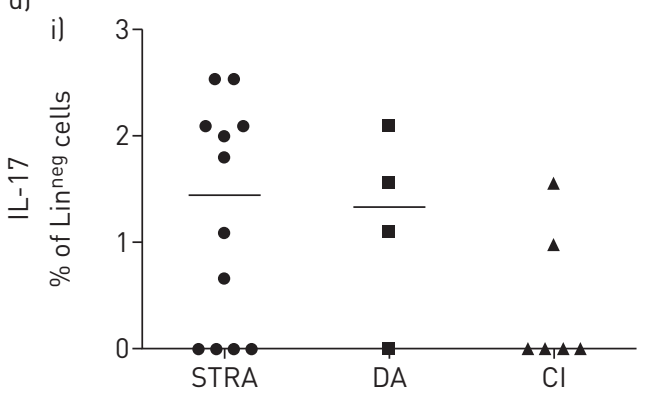

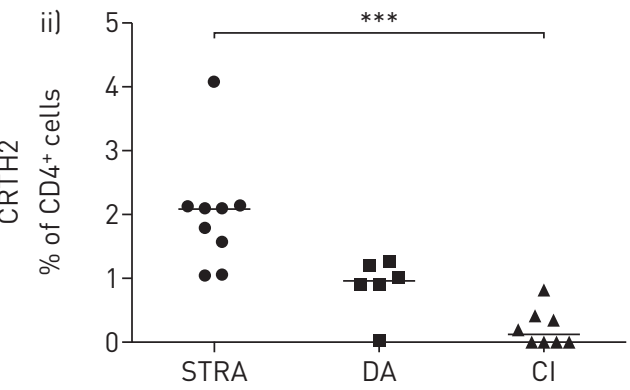
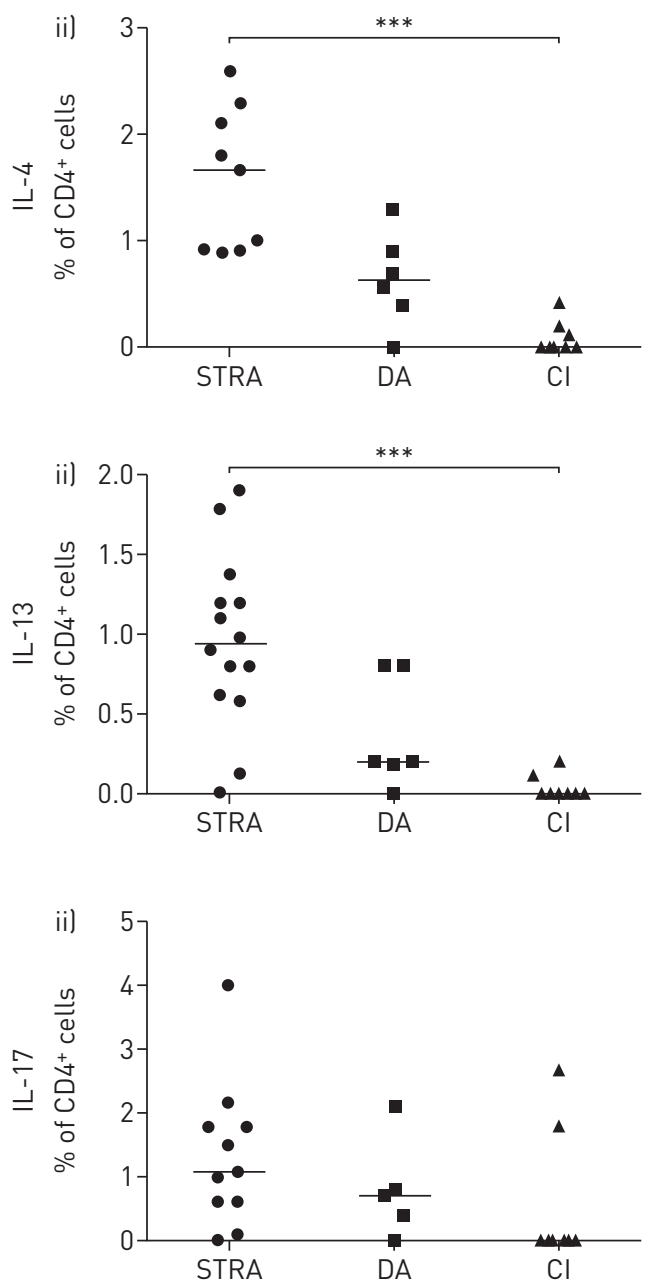

FIGURE 2 Sputum type-2 innate lymphoid cells (ILC2s) were higher in severe therapy-resistant asthma (STRA) than difficult asthma (DA), but interleukin (IL)-1 $17^{+}$cells were similar. a) Induced sputum frequency of i) $\mathrm{CRTH}^{+}$ILCs ( $\mathrm{Lin}^{\text {neg }} \mathrm{CD}_{4} 5^{+}$) and ii) $\mathrm{CD}^{+}$T-cells $\left(\mathrm{CD}^{+}{ }^{+} \mathrm{CD}^{+}\right)$from children with STRA, DA and with recurrent

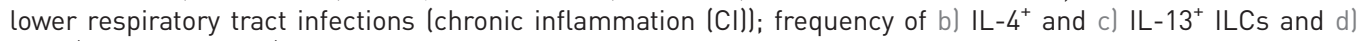
$\mathrm{IL}-17^{+} \mathrm{ILCs}$ and $\mathrm{CD} 4^{+}$T-cells. Kruskall-Wallis test with a Dunn's post-test, followed by Mann-Whitney test between indicated pairs of groups. ${ }^{*}: p<0.05,{ }^{* * *}: p<0.001$. STRA $n \geqslant 9$, DA $n \geqslant 4$ and $\mathrm{Cl} n \geqslant 6$.

similar manner, the majority of sputum $\mathrm{Lin}^{\text {neg }} \mathrm{CD} 45^{+} \mathrm{IL}-17^{+}$cells $(79 \%)$ did not express CD127. No Lin ${ }^{\text {neg }} \mathrm{CD} 45^{+} \mathrm{IL}-17^{+}$cells expressed the type 2 marker $\mathrm{CRTH} 2^{+}$(supplementary figure 5 ).

Airway type 2 lymphoid cells are reduced in STRA after systemic corticosteroids

To assess the clinical and immunological response to systemic steroids in children with STRA, sputum induction was performed before and 4 weeks after administration of intramuscular triamcinolone, as part of our clinical protocol [25] (figure 4a). Briefly, children with STRA had an assessment of 

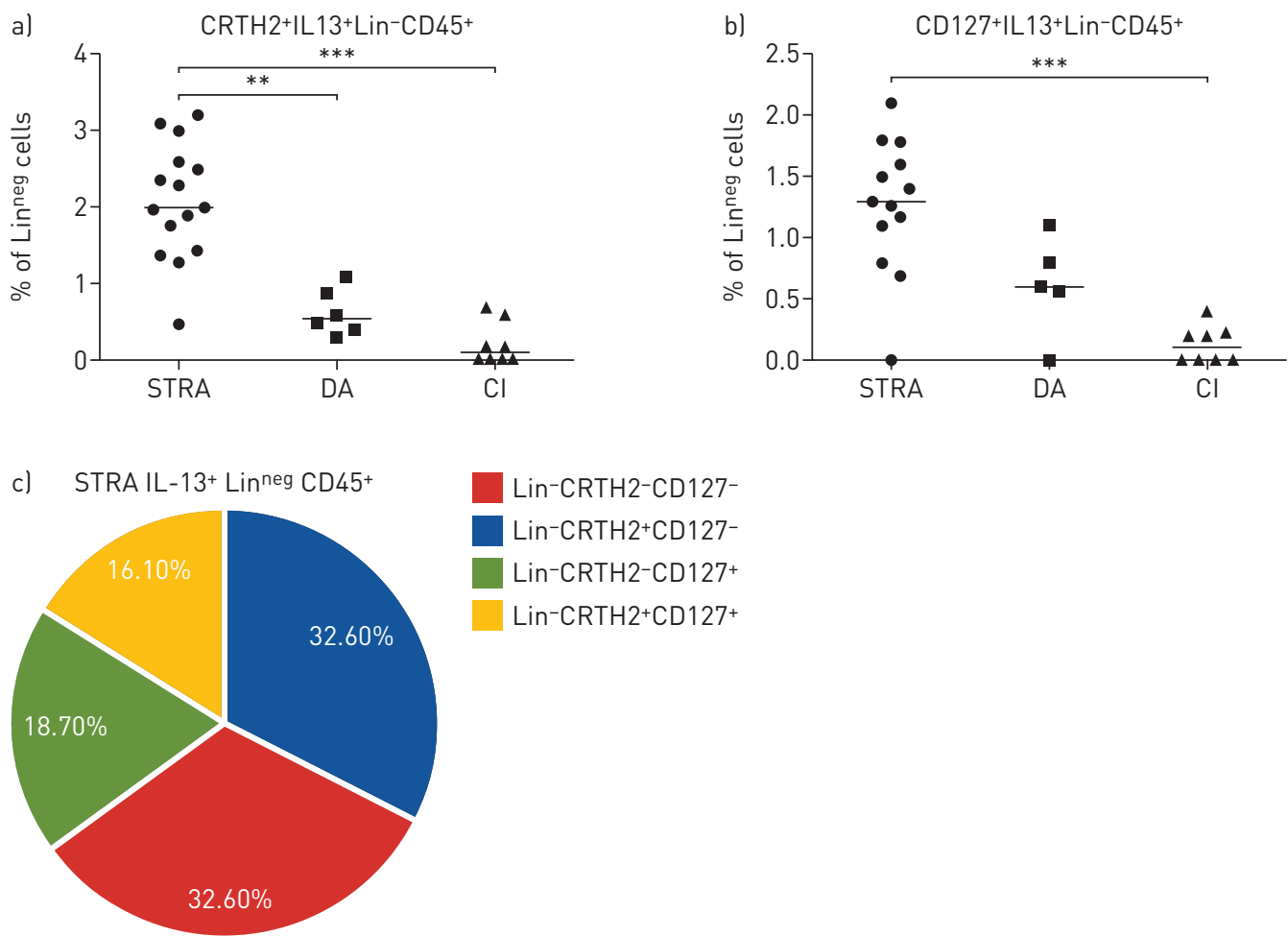

d)

CD127+CRTH2+IL13+Lin-CD45+

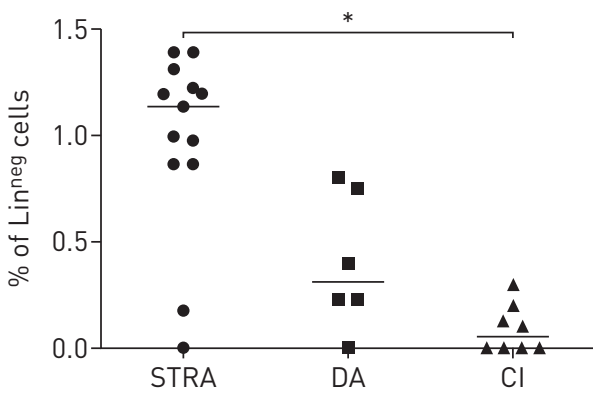

e)

CD127-CRTH2-IL13+Lin-CD45+

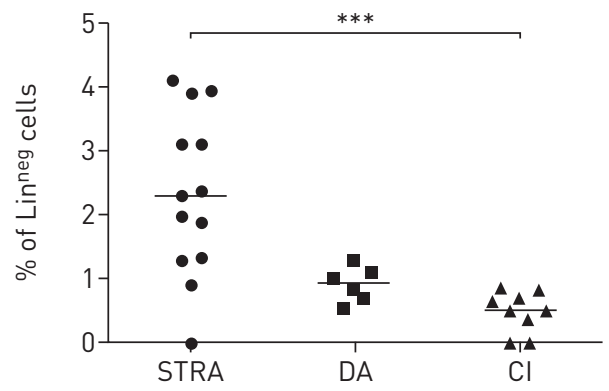

FIGURE 3 Phenotypic features of interleukin (IL)-13 ${ }^{+}$innate lymphoid cells (ILCs) in severe therapy-resistant asthma (STRA), difficult asthma (DA) and chronic inflammation (CI). Frequency of ILC2s $\left(I L-13^{+}\right.$Lin $\left.^{\text {neg }} \mathrm{CD} 45^{+}\right)$ expressing a) CRTH2 and b) CD127 in STRA, DA and $\mathrm{Cl}$ patient sputum. c) Frequencies of IL-13 ${ }^{+}$ILCs expressing CRTH2 and CD127 in sputum from STRA patients. Frequency of ILC2s $\left(I L-13^{+} L i n^{\text {neg }} C D 45^{+}\right)$ expressing d) both CRTH2 and CD127 and e) neither CRTH2 nor CD127 in STRA, DA and Cl patient sputum. Kruskall-Wallis test with a Dunn's post-test, followed by Mann-Whitney test between indicated pairs of groups. ${ }^{*}: p<0.05,{ }^{* *}: p<0.01,{ }^{* * *}: p<0.001$. STRA $n \geqslant 13, D A n \geqslant 5$ and $\mathrm{Cl} n \geqslant 8$.

spirometry, exhaled nitric oxide and symptom score on the morning of receiving triamcinolone and again 4 weeks later (figure 4a). There was no change in lung function (FEV1 \% predicted) up to 12 months after triamcinolone (figure $4 \mathrm{~b}$ ), but there was a significant reduction in exhaled nitric oxide fraction (figure 4c) and an improvement in symptoms assessed using the asthma control test 4 weeks later (figure $4 \mathrm{~d}$ ), and a reduction in asthma attacks (defined as short course of oral corticosteroids prescribed in the year after triamcinolone compared to the year before) (figure 4e), following administration of triamcinolone. The frequency of sputum eosinophils (figure 4f) and levels of sputum eosinophil peroxidase (a marker of eosinophil activation) were unchanged after triamcinolone (figure $4 \mathrm{~g}$ ). Detailed phenotyping of sputum lymphoid cell populations showed $\mathrm{Lin}^{\text {neg }} \mathrm{CD}_{4} 5^{+}$and $\mathrm{CD} 4^{+} \mathrm{T}$-cells expressing either $\mathrm{CRTH}_{2}{ }^{+}$or $\mathrm{IL}_{13}{ }^{+}$were reduced, suggesting that both of these cell types are steroid sensitive in the airways in vivo (figure 5a-d). In addition, quantification of type 2 mediators in sputum supernatants showed a reduction in both IL-13 and IL-5 after triamcinolone, even though eosinophil numbers remained elevated (figure 5e). 


$$
\begin{aligned}
& \text { a) } \\
& \text { Child } \\
& \text { with } \\
& \text { STRA }
\end{aligned}
$$

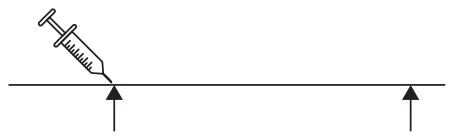

Visit 1

i.m. triamcinolone

Visit 2 4 weeks later
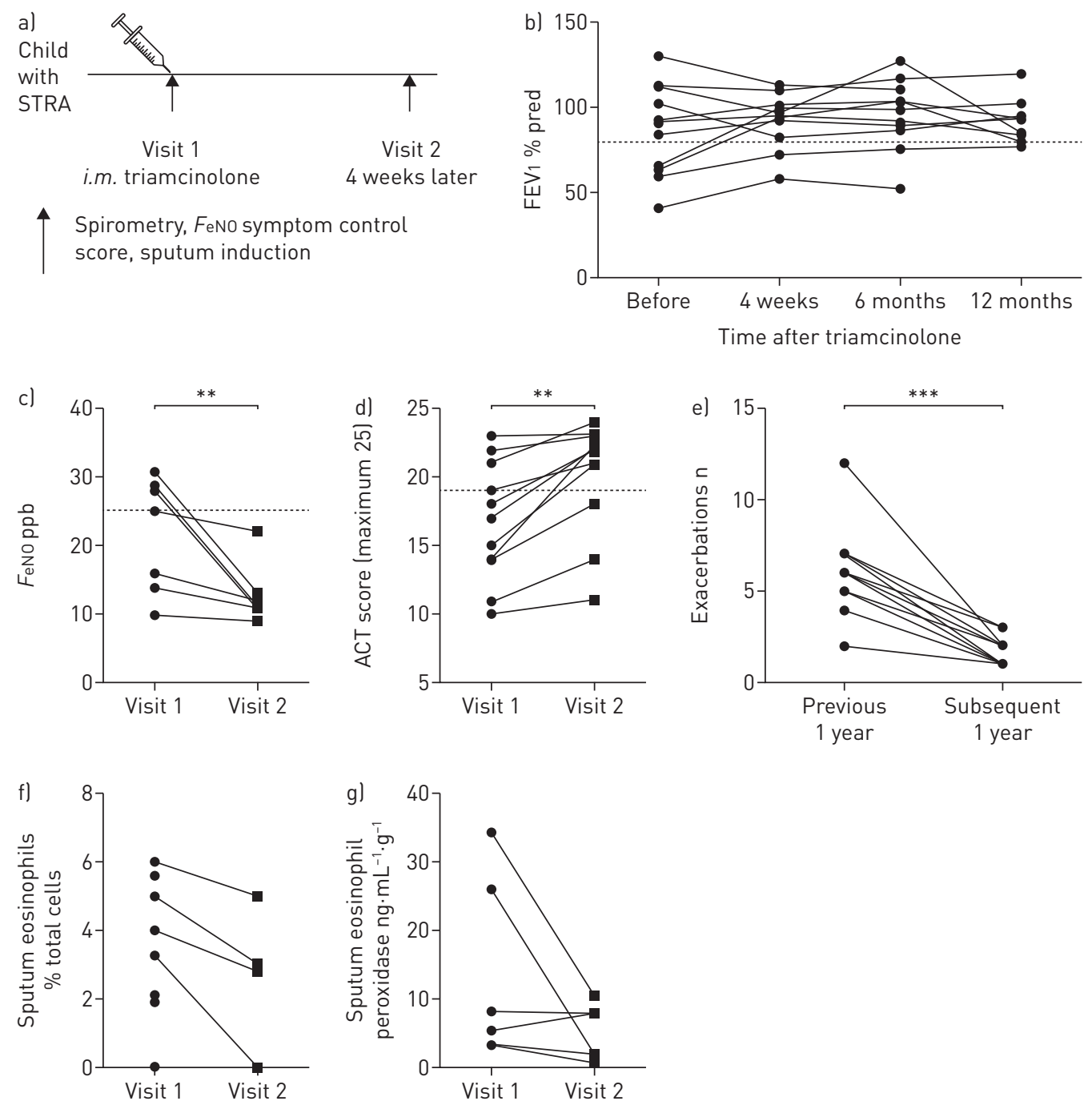

FIGURE 4 Reduction in clinical parameters after high-dose systemic steroids. a) Clinical outputs and immune phenotype of severe therapy-resistant asthma (STRA) patients were assessed before and 4 weeks after intramuscular treatment with the steroid triamcinolone. Clinical assessment of lung function via b) forced expiratory volume in $1 \mathrm{~s}\left(\mathrm{FEV}_{1}\right), \mathrm{c}$ ) exhaled nitric oxide fraction ( $\mathrm{FeNO}_{\mathrm{NO}}$, d) asthma control test (ACT) and e) frequency of clinical exacerbations (per year). Dashed lines show clinically normal FEV $(>80 \%), A C T(>19)$ and FeNO $(<25 \mathrm{ppb})(\mathrm{n}=11)$. f) Induced sputum eosinophil frequency $(n=5) ; \mathrm{g})$ levels of eosinophil peroxidase in sputum $(n=6)$. Mann-Whitney U-test. ${ }^{* *}$ : $p<0.01$.

Functional peripheral blood type 2 lymphoid cells defined using $\mathrm{IL}-13^{+}$are steroid sensitive in vitro To test the hypothesis that ILCs do not just respond to a muted inflammatory environment resulting from steroid suppression in vivo, but are themselves steroid sensitive, we cultured PBMCs isolated from nine STRA patients sensitised to HDM. Cells were stimulated with HDM extract with or without budesonide and then ILC and T-cell subsets were analysed. Stimulation with HDM resulted in a significant increase in Lin ${ }^{\text {neg }} \mathrm{CD} 45^{+} \mathrm{IL}-13^{+}$ILCs and $\mathrm{CD} 4^{+} \mathrm{IL} 13^{+}$T-cells, in PBMC cultures from HDM-sensitised STRA children (figure $6 \mathrm{a}, \mathrm{b})$. However, there was a significant reduction in the number of IL-13 ${ }^{+}$ILCs and IL13 ${ }^{+}$T-cells when the PBMCs were cultured with HDM and budesonide (figure 6a,b). In addition, levels of secreted IL-13 following HDM stimulation in PBMC cultures were reduced by budesonide (figure 6c).

\section{Circulating IL-17+ ILCs and Th17 cells are steroid refractory}

To determine the effect of steroid treatment on IL-17 expressing lymphoid cells, we examined Lin ${ }^{\text {neg }}{ }^{C D} 45^{+}$ILCs and $\mathrm{CD}^{+}$cells in PBMC cultures from HDM-sensitised patients (n=9) with STRA. Intriguingly, we found that budesonide alone resulted in a significant induction of IL- $17^{+}$ILCs, while IL-17 ${ }^{+}$T-cells remained unchanged (figure 7a,b). However, no difference was noted in levels of IL-17 protein in the supernatant following addition of either HDM and/or budesonide (figure 7c). 
Airway ILCs
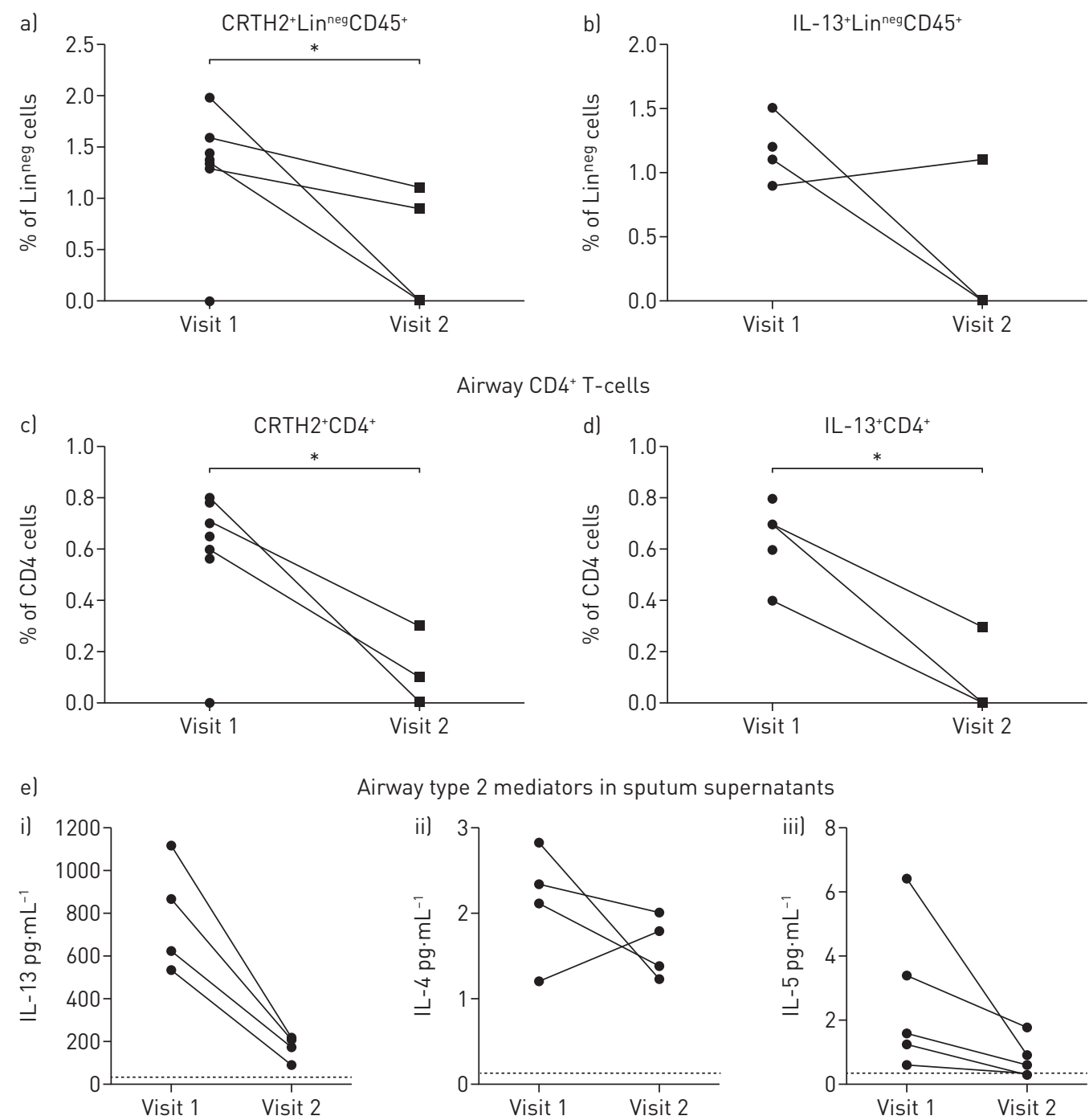

Airway type 2 mediators in sputum supernatants
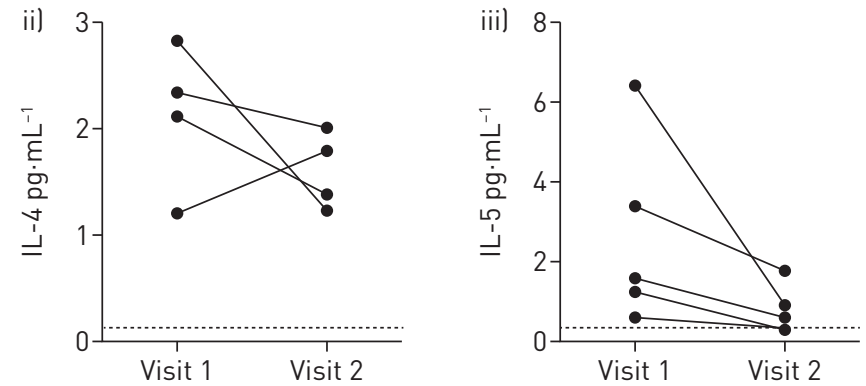

FIGURE 5 Airway innate lymphoid cells (ILCs) and T-cells are steroid sensitive in vivo, after high-dose systemic steroids. Induced sputum frequency of ILCs ( $\left(\right.$ Lin $\left.^{\text {neg }} \mathrm{CD} 45^{+}\right)$and $\mathrm{CD} 4^{+}$T-cells $\left(\mathrm{CD} 4^{+} \mathrm{CD} 3^{+}\right)$expressing a,c) CRTH2 or b,d) interleukin (IL)-13. e) i) IL-13, ii) IL-4 and iii) IL-5 levels in sputum supernatant. $\mathrm{n} \geqslant 3$. Mann-Whitney U-test. *: p<0.05.
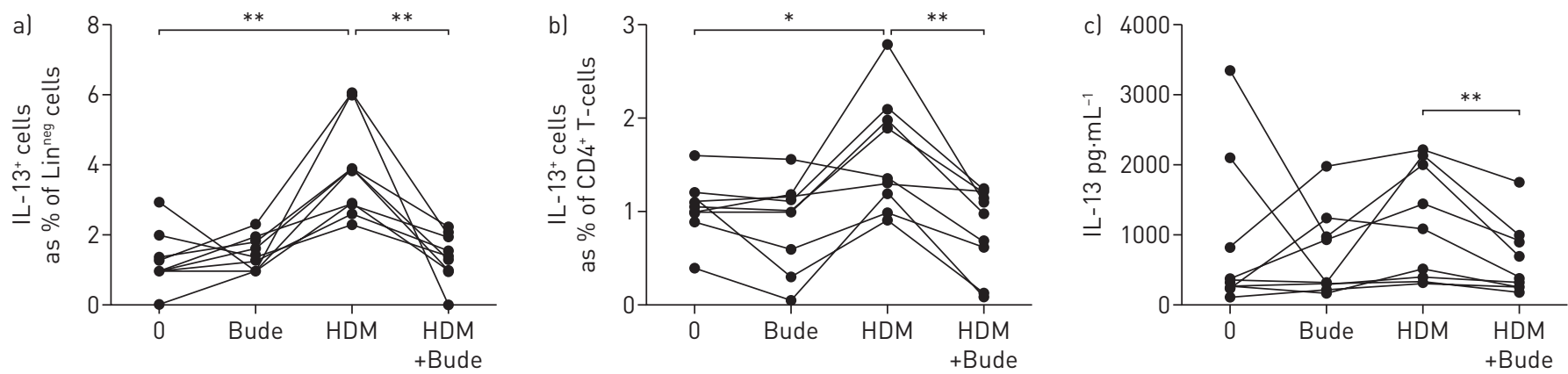

FIGURE 6 Frequency of interleukin (IL)- $13^{+} \mathrm{CD} 4^{+} \mathrm{T}$-cells and IL-13+ innate lymphoid cells (ILCs) from severe therapy-resistant asthma (STRA) patient peripheral blood are significantly reduced after steroids in vitro. Frequency of IL-13 expressing a) ILC2s (CD127 ${ }^{+}$CD $45^{+}$Lin ${ }^{\text {neg }}$ ) and b) CD4 T-cells (CD $\left.45^{+} \mathrm{CD}^{+} \mathrm{CD}^{+}\right)$after in vitro treatment of peripheral blood mononuclear cell cultures with house dust mite (HDM) and/or budesonide (Bude) for 72 h. c) IL-13 protein levels in culture supernatants. STRA $n=9$. Wilcoxon matched pairs test. *: $p<0.05,{ }^{* *}: p<0.01$. 

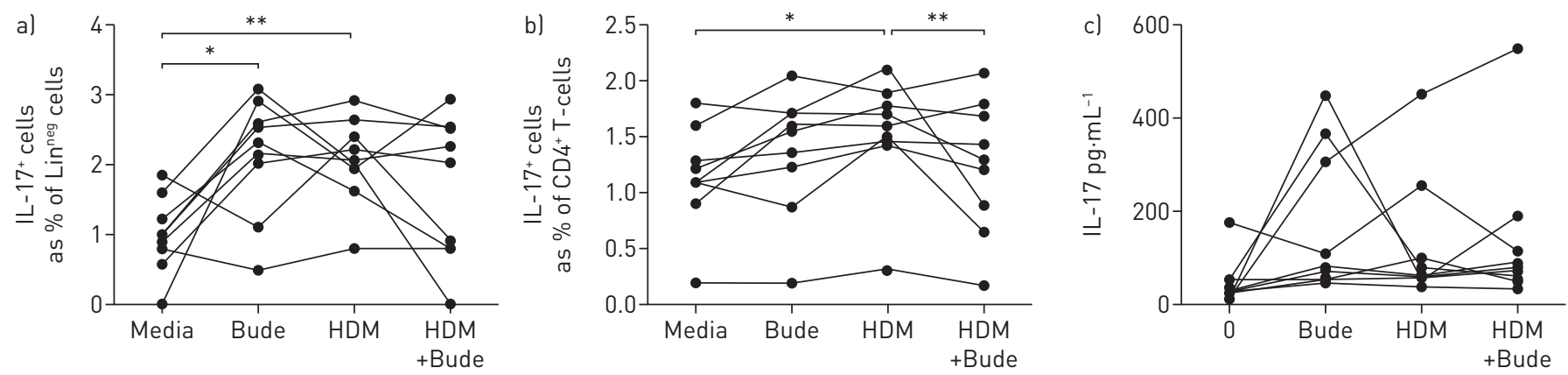

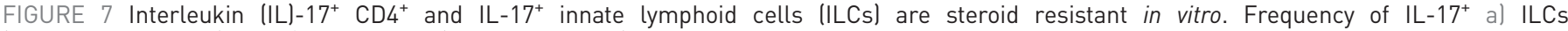
$\left(\mathrm{CD} 127^{+} \mathrm{CD} 45^{+} \mathrm{Lin}^{\text {neg }}\right)$ and b) $\mathrm{CD} 4 \mathrm{~T}$-cells $\left(\mathrm{CD} 45^{+} \mathrm{CD} 3^{+} \mathrm{CD} 4^{+}\right)$after in vitro treatment of peripheral blood mononuclear cells cultures with house dust mite (HDM) and/or budesonide (Bude) for $72 \mathrm{~h}$; c) IL-17 protein levels in culture supernatants. ILC cultures $\mathrm{n}=8$. Wilcoxon matched pairs test. $*$ : $p<0.05,{ }^{* *}: p<0.01$.

\section{IL-13+ innate lymphoid cells respond directly to steroids}

In order to establish whether a pure population of ILCs $\left(\mathrm{CD} 45^{+}, \mathrm{Lin}^{\text {neg }}, \mathrm{CD} 161^{+}, \mathrm{CD} 127^{+}, \mathrm{CRTH2} 2^{+}\right.$ and $\mathrm{C}-\mathrm{Kit}^{\mathrm{var}}, \mathrm{IL}_{-} 13^{+}$) responded directly to steroid treatment, peripheral blood cells were sorted and cultured with recombinant IL-2, IL-7 and IL-33 to skew towards a type 2 phenotype [24], for $\geqslant 4$ weeks (>99\% ILC2s). ILCs were sorted from adults because of ethical restrictions preventing large volumes of blood being obtained from children to isolate this rare cell population. Incubation of in vitro cultured cells with budesonide resulted in a reduction in the number of ILCs expressing IL-13, together with reduced IL-13 protein levels in culture supernatants (figure 8a,b). ILCs, which are not antigen specific, but may respond to one of the complex HDM components such as lipids or proteins, did not respond directly to HDM stimulation (figure 8a,b). In addition, in the presence of budesonide, IL-13 gene

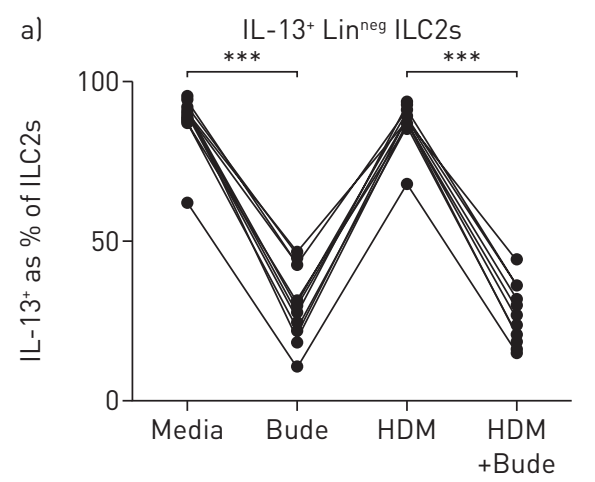

b)

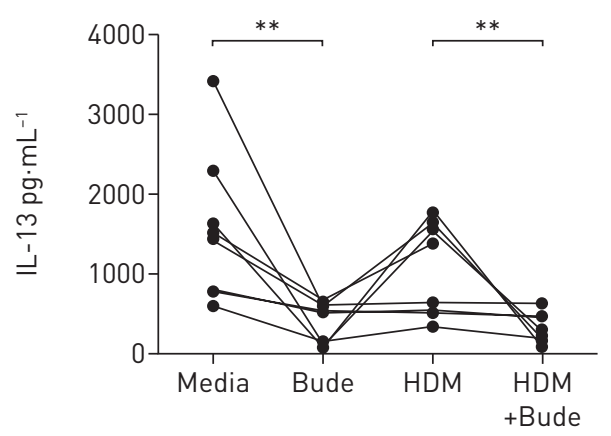

c)

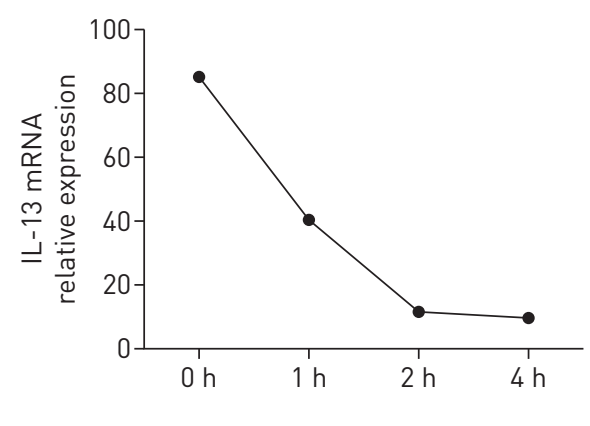

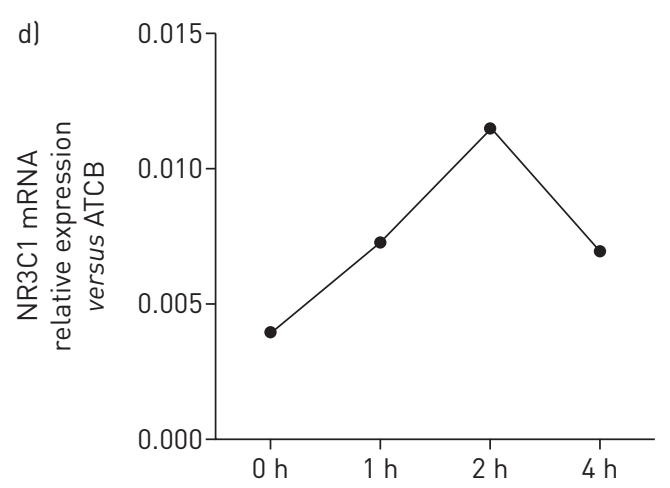
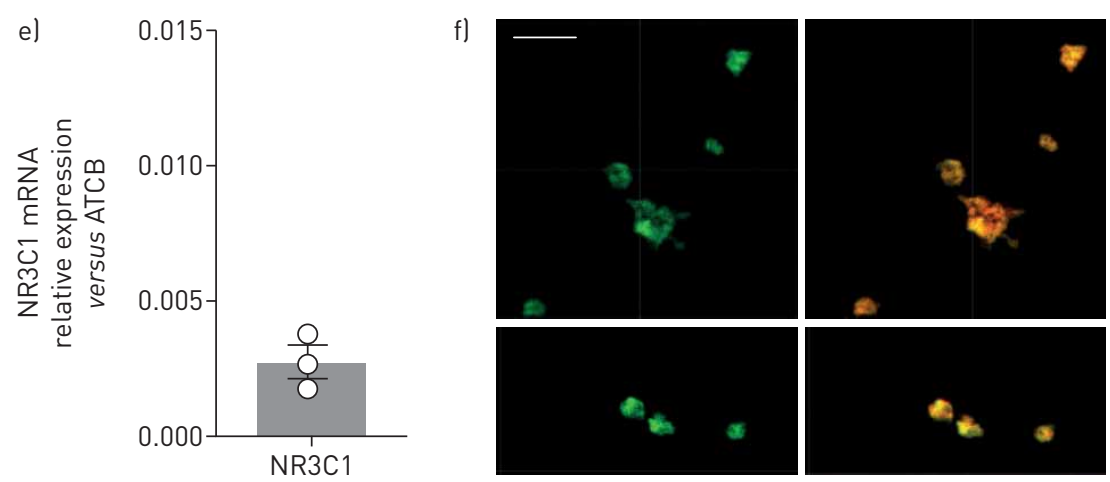

FIGURE 8 Cultured type 2 innate lymphoid cells (ILC2s) are steroid sensitive in vitro. Expression of a) interleukin (IL)-13 by ILC cultures ( $\mathrm{Lin}{ }^{\text {neg }} \mathrm{CD} 45^{+} \mathrm{CD} 161^{+} \mathrm{CRTH} 2^{+} \mathrm{CD} 127^{+}$) treated with house dust mite (HDM) and/or budesonide (Bude); b) IL-13 protein levels in ILC culture supernatants. mRNA expression of c) IL13 and d) NR3C1 at baseline and following incubation of cells with budesonide for up to $4 \mathrm{~h}$; e) ILC2s were enriched and sorted $\left(\mathrm{Lin}^{\text {neg }} \mathrm{CD} 45^{+} \mathrm{CD} 161^{+} \mathrm{CRTH} 2^{+} \mathrm{CD} 127^{+}\right)$from peripheral blood of three individual donors. mRNA expression of $\mathrm{NR} 3 \mathrm{C} 1$ relative to endogenous controls was determined. f) Immunofluoresence staining of the glucocorticoid receptor and overlaid with actin (red) on human ILC cultures. Scale bar $=20 \mu \mathrm{m}$. ILC cultures $\mathrm{n}=8$. Wilcoxon matched pairs test. ${ }^{* *}: p<0.01,{ }^{* * *}: p<0.001$. 
expression was rapidly downregulated (figure 8c). Furthermore, we looked for expression of the glucocorticoid receptor and showed constitutive expression of NR3C1 by ILCs, with levels of expression increasing in the presence of budesonide (figure 8d). Expression of the glucocorticoid receptor on purified ILCs at the protein level was confirmed by immunofluorescence (figure 8f). These data demonstrate definitively that in the presence of sufficient doses of steroids, functional $\mathrm{Lin}^{\text {neg }} \mathrm{CD} 45^{+} \mathrm{IL}-13^{+}$are steroid sensitive.

\section{Discussion}

Although paediatric STRA is recognised as being characterised by severe atopy and steroid-resistant airway eosinophilia [1, 25], little was known about lymphoid cell populations driving the disease. We have shown increased numbers of ILCs, expressing the type 2 markers CRTH2 and IL-13 in the airways from STRA children during stable disease compared to children with DA and disease control patients. Airway type 2 ILCs, T-cells and eosinophils were elevated in STRA despite prior assessments to ensure optimal adherence to high-dose maintenance inhaled steroid therapy. In contrast, neither lymphoid populations nor eosinophils were elevated in the airways of DA children, suggesting that these are clinically and molecularly distinct phenotypes and DA is characterised by more steroid-sensitive disease. Contrary to our hypothesis, we found that although airway CRTH2 $2^{+} \mathrm{IL}-13^{+}$ILCs were increased in STRA at baseline, they were reduced by high-dose systemic steroids in vivo and steroid stimulation in vitro. Of note, none of the children were on maintenance oral steroids. In contrast, IL- $17^{+}$ILCs were similar in STRA, DA and controls, and were steroid resistant in vitro. There was a reduction in numbers of airway type 2 lymphoid cells following high-dose systemic steroids and an improvement in symptoms and asthma attacks, but no change in eosinophil numbers in STRA. However, systemic steroids are not a feasible long-term therapeutic option and alternative steroid-sparing therapies that dampen type 2 lymphoid cells are needed.

We acknowledge the numbers of children from whom good quality samples to phenotype sputum lymphoid cells could be obtained is small. We did not select a subgroup for whom data are shown in any of the figures. The reason the numbers in some of the figures is low is because of variable cell numbers in the sputum and limited paired sputum samples before and after triamcinolone, and this is an inevitable weakness of these sorts of studies. We did not select specific children to include in the results. However, we did clinically phenotype the children very carefully and ensured that objective assessments of adherence were undertaken prior to defining STRA and DA, and the findings show clear distinctions between the groups despite small numbers.

We have previously reported the importance of distinguishing children with DA, who have poor control because of underlying modifiable factors, such as poor adherence, from those with STRA, who remain with poor control despite optimal adherence $[4,5]$. We have demonstrated clinical distinctions between STRA and DA, whereby STRA is characterised by persistent poor lung function, frequent attacks and severe and multiple atopic sensitisation [6]. However, the molecular phenotypes of DA and STRA were unknown. We now demonstrate that children with true severe asthma (STRA) have elevated airway eosinophils, IL-13 ${ }^{+}$ ILCs and T-cells compared to children with DA, and importantly these distinctions are only apparent in sputum, not in peripheral blood. Our data have highlighted the importance of investing time and resources to accurately clinically phenotype children with poor asthma control despite maximal prescribed therapy in order to distinguish STRA from DA. If this is not done, as increasing targeted biologics and small molecule therapies become available [26], there is a risk that children may be given these novel drugs inappropriately.

Although airway ILCs are increased in STRA, their role in promoting disease severity, particularly in humans, remains unclear. Since IL-33 is increased in STRA, is a relatively steroid-resistant cytokine [10] and induces ILCs [12], we undertook detailed phenotyping of airway ILCs and their response to steroids. We have shown that although the extracellular marker CRTH2 is used to denote type 2 lymphoid cells, and CD127 is used to denote ILCs, the majority ( $\sim 65 \%)$ of airway IL- $13^{+} \mathrm{Lin}^{\text {neg }} \mathrm{CD} 45^{+}$cells are CD $127^{-}$ and approximately half are $\mathrm{CRTH}_{2}{ }^{-}$. However, the majority of human studies to date that have reported on the prevalence and response of airway ILCs to steroids in the context of allergic disease have included extracellular markers as part of the definition [27]. This suggests that a large proportion of cells that are lymphoid in origin and are lineage negative, but have the capacity to secrete type 2 cytokines, and are therefore functionally equivalent to an ILC2, have been disregarded. We have shown here, and confirmed our previous findings [17], that in the airways of children with severe asthma, a significant proportion of $\mathrm{Lin}^{-} \mathrm{CD} 45^{+}$cells do not express the extracellular markers currently used to define ILC2s. It is essential to consider the plasticity of ILCs and the influence of the local environment on their function [28]. Restricting the definition to include the extracellular markers CD127 and/or CRTH2 may therefore be inappropriate for describing ILC2 function, particularly those from tissues in children.

We report that ILCs $\left(\mathrm{Lin}^{\text {neg }} \mathrm{CD} 5^{+} \mathrm{CRTH} 2^{+}\right.$and $\mathrm{Lin}^{\text {neg }}{ }^{\mathrm{CD}} 45^{+} \mathrm{IL}-13^{+}$) are sensitive to steroids both in vivo and in vitro. These results mirrored the response of Th2 cells to steroids. The data are in agreement with 
studies in nasal polyps of adults with chronic rhinosinusitis which have shown that ILC2s are steroid sensitive [29]. However, this is the first demonstration in patients and in airway ILCs following steroids in severe asthma. It has previously been reported that ILC2s in BAL fluid from adult patients with asthma were resistant to dexamethasone [24]. However, that was demonstrated by in vitro stimulation of airway ILCs using a steroid that is not routinely used in clinical practice to treat asthma. We have maximised information obtained from our routine clinical protocol which involves a single dose of systemic steroids in children with STRA [25], and shown that sputum IL- $13^{+}$ILCs and CRTH2 ${ }^{+}$ILCs reduced significantly, in tandem with $\mathrm{CD}^{+}$type 2 lymphocytes following triamcinolone. In order to confirm the effect of steroids on type 2 ILCs alone, and to eliminate the effects of other leukocytes, we demonstrated cultures of peripheral blood type 2 ILCs had glucocorticoid receptor expression and also reduced when stimulated with budesonide in vitro. It is plausible that although ILC2s are steroid sensitive, delivery of currently available inhaled steroid medications does not reach the airways at optimal concentrations to allow a reduction in the number of these cells, thus explaining elevated numbers in STRA during stable disease. The reduction in exacerbations, coupled with improved symptom score and the associated reduction in type 2 lymphoid cells following triamcinolone, suggest that it is likely that these cells do play a role in driving symptoms in children with STRA despite high-dose treatment with inhaled steroids.

Although we have recently shown no increase in airway Th17 cells in paediatric STRA [30], we had not previously investigated the potential role of IL- $17^{+}$ILCs. In agreement with our findings of Th17 cells, airway IL- $17^{+}$ILCs were not increased in STRA compared to DA or disease controls. Furthermore, unlike type 2 lymphoid cells, IL-17 $7^{+}$ILCs and Th17 cells were steroid resistant both in vivo and in vitro. In addition, we have demonstrated a significant induction of IL- $17^{+}$ILCs with budesonide in vitro. The Th17/IL-17 axis has been linked to severe, steroid-resistant adult asthma [31]; however, the number of IL- $17^{+}$cells in our patients with STRA did not show any correlation with clinical parameters. This is in keeping with our previous report of a lack of correlation between IL-17 levels, Th17 cells and clinical features in paediatric STRA [30]. Even though the disease control children with recurrent infections had an airway neutrophilia, they did not have increased IL- $17^{+}$lymphoid cells. This may be because the majority of lymphoid cells in children with neutrophilic airways diseases are in the airway wall, not the lumen $[32,33]$.

We accept that the number of children in the cohort studied before and after triamcinolone was small; this is because not all were able to produce an adequate sputum sample at both time points as this is a technically difficult procedure in young children. However, despite the small numbers, the data demonstrate clear changes in type2 lymphoid cells, and no previous studies have demonstrated longitudinal changes in rare cell populations in airway samples from children. Importantly, all children were clinically stable at the time of sputum induction, with $\geqslant 2$ weeks since any previous exacerbation. The lack of change in lung function and the relatively high values at baseline for FEV 1 may be explained by the "stable" nature of the patients, but is a consistent clinical finding in children with severe asthma [34]. It would be interesting to assess any change in proportions of ILCs during exacerbation. Murine data suggest that ILC3s are important in exacerbations during obese allergic airways disease [35] and ILC2s contribute to influenza-induced episodes [36]. However, their role in virally induced exacerbations in humans remains uncertain.

Significant strengths of our data are the inclusion of carefully clinically characterised patients with STRA and DA, and the utility of non-invasive airway sputum cells to demonstrate changes in immune cells after steroid treatment in vivo and in vitro. We accept our study has limitations. We included children with recurrent lower respiratory tract infections, i.e. chronic lower airway inflammation as the best pragmatic control group. Ideally, healthy controls should be recruited; however, obtaining airway samples from healthy children is not ethically possible. Of note, previous adult studies have also included similar types of disease controls $[24,37]$. In addition, even though significantly more disease controls had neutrophils in sputum, this did not impact numbers of IL- $17^{+}$lymphoid cells, even though IL-17 has been associated with neutrophilia $[38,39]$.

In summary, we have shown the contribution of airway $\mathrm{Lin}^{\text {neg }} \mathrm{CD} 45^{+} \mathrm{CRTH} 2^{+}$and $\mathrm{Lin}{ }^{\text {neg }} \mathrm{CD} 45^{+} \mathrm{IL}-13^{+}$ ILCs in children with STRA compared to DA, and the effect of steroids on these cells in paediatric STRA. The reduction in both type 2 ILCs and T-cells after systemic steroids was associated with a reduction in exacerbations and improvement in symptoms. However, there was no change in numbers of airway eosinophils following steroids. We have also shown that IL- $17^{+}$ILCs and Th17 cells were not increased in STRA compared to DA at baseline and both cell types persisted despite steroids. The clinical improvement despite elevated IL- $17^{+}$cells questions their role in mediating the pathophysiology of paediatric STRA.

Acknowledgements: The authors thank Jane Srivastava and Jessica Rowley, of the Imperial College Core Flow Cytometry facility (London, UK), for assistance with cell sorting. We would also like to thank Robert Oliver and Helen Stoelting 
(Imperial College London, London, UK) for their help in generating the quantitative PCR data and Lucy Robson (Imperial College London) for culturing the ILC cultures.

Support statement: This study was supported by grants from the Wellcome Trust UK (107059/Z/15/Z) and Asthma UK (AUK-IG-2014-269). C.M. Lloyd is a Wellcome Senior Fellow, A. Bush is an NIHR Senior Investigator and S. Saglani is an NIHR Career Development Fellow (CDF-2014-07-019). Funding information for this article has been deposited with the Crossref Funder Registry.

Conflict of interest: F. Puttur has nothing to disclose. L.G. Gregory has nothing to disclose. L. Denney has nothing to disclose. L. Fleming reports other funding from GSK, Sanofi, Novartis, Boehrringer Ingelheim and Astra Zeneca, outside the submitted work. A. Bush has nothing to disclose. C.M. Lloyd has nothing to disclose. S. Saglani has nothing to disclose. P. Nagakumar has nothing to disclose.

\section{References}

1 Bossley CJ, Fleming L, Gupta A, et al. Pediatric severe asthma is characterized by eosinophilia and remodeling without $\mathrm{T}_{\mathrm{H}} 2$ cytokines. J Allergy Clin Immunol 2012; 129: 974-982.

2 Chung KF, Wenzel SE, Brozek JL, et al. International ERS/ATS guidelines on definition, evaluation and treatment of severe asthma. Eur Respir J 2014; 43: 343-373.

3 Chung KF, Wenzel S, European Respiratory Society/American Thoracic Society Severe Asthma International Guidelines Task Force. From the authors: International European Respiratory Society/American Thoracic Society guidelines on severe asthma. Eur Respir J 2014; 44: 1378-1379.

$4 \quad$ Bush A, Saglani S. Management of severe asthma in children. Lancet 2010; 376: 814-825.

5 Bush A, Fleming L, Saglani S. Severe asthma in children. Respirology 2017; 22: 886-897.

6 Sharples J, Gupta A, Fleming L, et al. Long-term effectiveness of a staged assessment for paediatric problematic severe asthma. Eur Respir J 2012; 40: 264-267.

7 Fitzpatrick AM, Jackson DJ, Mauger DT, et al. Individualized therapy for persistent asthma in young children. J Allergy Clin Immunol 2016; 138: 1608-1618.

8 Fahy JV. Type 2 inflammation in asthma - present in most, absent in many. Nat Rev Immunol 2015; 15: 57-65.

9 Brusselle GG, Maes T, Bracke KR. Eosinophils in the spotlight: eosinophilic airway inflammation in nonallergic asthma. Nat Med 2013; 19: 977-979.

10 Saglani S, Lui S, Ullmann N, et al. IL-33 promotes airway remodeling in pediatric patients with severe steroid-resistant asthma. J Allergy Clin Immunol 2013; 132: 676-685.

11 Castanhinha S, Sherburn R, Walker S, et al. Pediatric severe asthma with fungal sensitization is mediated by steroid-resistant IL-33. J Allergy Clin Immunol 2015; 136: 312-322.

12 Mjösberg JM, Trifari S, Crellin NK, et al. Human IL-25- and IL-33-responsive type 2 innate lymphoid cells are defined by expression of CRTH2 and CD161. Nat Immunol 2011; 12: 1055-1062.

13 Kim HY, Chang YJ, Subramanian S, et al. Innate lymphoid cells responding to IL-33 mediate airway hyperreactivity independently of adaptive immunity. J Allergy Clin Immunol 2012; 129: 216-227.

14 Barlow JL, Peel S, Fox J, et al. IL-33 is more potent than IL-25 in provoking IL-13-producing nuocytes (type 2 innate lymphoid cells) and airway contraction. J Allergy Clin Immunol 2013; 132: 933-941.

15 Spits H, Di Santo JP. The expanding family of innate lymphoid cells: regulators and effectors of immunity and tissue remodeling. Nat Immunol 2011; 12: 21-27.

16 Smith SG, Chen R, Kjarsgaard M, et al. Increased numbers of activated group 2 innate lymphoid cells in the airways of patients with severe asthma and persistent airway eosinophilia. J Allergy Clin Immunol 2016; 137: 75-86.

17 Nagakumar P, Denney L, Fleming L, et al. Type 2 innate lymphoid cells in induced sputum from children with severe asthma. J Allergy Clin Immunol 2016; 137: 624-626.

18 Molet S, Hamid Q, Davoine F, et al. IL-17 is increased in asthmatic airways and induces human bronchial fibroblasts to produce cytokines. J Allergy Clin Immunol 2001; 108: 430-438.

19 Gupta A, Dimeloe S, Richards DF, et al. Defective IL-10 expression and in vitro steroid-induced IL-17A in paediatric severe therapy-resistant asthma. Thorax 2014; 69: 508-515.

20 Bush A, Saglani S, Fleming L. Severe asthma: looking beyond the amount of medication. Lancet Respir Med 2017; 5: 844-846.

21 Hurst SD, Muchamuel T, Gorman DM, et al. New IL-17 family members promote Th1 or Th2 responses in the lung: in vivo function of the novel cytokine IL-25. J Immunol 2002; 169: 443-453.

22 Yancey SW, Ortega HG, Keene ON, et al. Meta-analysis of asthma-related hospitalization in mepolizumab studies of severe eosinophilic asthma. J Allergy Clin Immunol 2017; 139: 1167-1175.

23 Ullmann N, Bossley CJ, Fleming L, et al. Blood eosinophil counts rarely reflect airway eosinophilia in children with severe asthma. Allergy 2013; 68: 402-406.

24 Liu S, Verma M, Michalec L, et al. Steroid resistance of airway type 2 innate lymphoid cells from patients with severe asthma: the role of thymic stromal lymphopoietin. J Allergy Clin Immunol 2018; 141: 257-268.

25 Bossley CJ, Fleming L, Ullmann N, et al. Assessment of corticosteroid response in pediatric patients with severe asthma by using a multidomain approach. J Allergy Clin Immunol 2016; 138: 413-420.

26 Diver S, Russell RJ, Brightling CE. New and emerging drug treatments for severe asthma. Clin Exp Allergy 2018; 48: 241-252.

27 Chen R, Smith SG, Salter B, et al. Allergen-induced increases in sputum levels of group 2 innate lymphoid cells in subjects with asthma. Am J Respir Crit Care Med 2017; 196: 700-712.

28 Silver JS, Kearley J, Copenhaver AM, et al. Inflammatory triggers associated with exacerbations of COPD orchestrate plasticity of group 2 innate lymphoid cells in the lungs. Nat Immunol 2016; 17: 626-635.

29 Walford HH, Lund SJ, Baum RE, et al. Increased ILC2s in the eosinophilic nasal polyp endotype are associated with corticosteroid responsiveness. Clin Immunol 2014; 155: 126-135.

30 Andersson CK, Adams A, Nagakumar P, et al. Intraepithelial neutrophils in pediatric severe asthma are associated with better lung function. J Allergy Clin Immunol 2017; 139: 1819-1829. 
31 Chesné J, Braza F, Mahay G, et al. IL-17 in severe asthma. Where do we stand? Am J Respir Crit Care Med 2014; 190: 1094-1101.

32 Regamey N, Tsartsali L, Hilliard TN, et al. Distinct patterns of inflammation in the airway lumen and bronchial mucosa of children with cystic fibrosis. Thorax 2012; 67: 164-170.

33 Tan HL, Regamey N, Brown S, et al. The Th17 pathway in cystic fibrosis lung disease. Am J Respir Crit Care Med 2011; 184: 252-258.

34 Fitzpatrick AM, Moore WC. Severe asthma phenotypes - how should they guide evaluation and treatment? J Allergy Clin Immunol Pract 2017; 5: 901-908.

35 Everaere L, Ait-Yahia S, Molendi-Coste O, et al. Innate lymphoid cells contribute to allergic airway disease exacerbation by obesity. J Allergy Clin Immunol 2016; 138: 1309-1318.

36 Li BWS, de Bruijn MJW, Lukkes M, et al. T cells and ILC2s are major effector cells in influenza-induced exacerbation of allergic airway inflammation in mice. Eur J Immunol 2019; 49: 144-156.

37 Doe C, Bafadhel M, Siddiqui S, et al. Expression of the T helper 17-associated cytokines IL-17A and IL-17F in asthma and COPD. Chest 2010; 138: 1140-1147.

38 Marzano AV, Borghi A, Wallach D, et al. A comprehensive review of neutrophilic diseases. Clin Rev Allergy Immunol 2018; 54: 114-130.

39 Allen JE, Sutherland TE, Rückerl D. IL-17 and neutrophils: unexpected players in the type 2 immune response. Curr Opin Immunol 2015; 34: 99-106. 